\title{
INFLUENCE OF ROTATION ON THE STELLAR PARAMETERS
}

\author{
V. WENSKE AND D. SCHÖNBERNER \\ Institut für Theor. Physik und Sternwarte der Universität Kiel \\ Olshausenstr. 40, W-2300 Kiel, Germany
}

For several years it has become quite common to derive stellar parameters like effective temperature, $T_{\text {eff }}$, and surface acceleration, $g$, by means of properly calibrated photometric indices, and to use these values for the derivation of important properties of stellar aggregates (viz. ages, star formation history, distances, etc.). Photometric observations, however, fail to give informations about one important property of a star: its rotational rate!

The main effect of rotation is to increase the size of the star, mainly in the equatorial region, leading to lower surface temperatures and accelerations. Since in non-spherical stars $T_{\text {eff }}$ and $g$ depend on the latitude, the observed values, which are, of course, averages over the visible hemisphere, depend on the angle of inclination, $i$. Collins \& Sonneborn (1977) utilized the rigidly rotating stellar models of Sackmann and Anand (1970) to compute emergent fluxes and photometric indices for various stellar masses, inclination angles $i$ and rotational parameters $w=\frac{\omega}{\omega_{\text {crit }}}, \omega_{\text {crit }}$ being the break-up rotational rate. These indices, viz. $c_{0}$ and $\beta$, represent then averages over the visible part of the rotally distorted stellar models. Closer inspections indicated also that emergent fluxes and line profiles of rotationally distorted stars can be matched by the predictations of standard model atmospheres to a very high accuray, even for $w$ close to unity (Wenske 1992, Diplom Thesis).

We observed nearly all B-type stars in the two open clusters NGC 2264 and $\alpha \operatorname{Per}(\mathrm{Mel} 20)$. The spectrogrammes, covering $\approx 130 \AA$, centered at $H_{\gamma}$, were obtained with the Coudé spectrograph (with CCD) of the $2.2 \mathrm{~m}$ telescope at the Calar Alto observatory. The resolution is $\approx 0.25 \AA$, and $\mathrm{S} / \mathrm{N}$ better than 50 in most cases. These observations were supplemented by $u v b y \beta$ photometry from the literature, and by IUE low-resolution spectrogrammes if available.

First we determined the effective temperatures from the photometry according to the prescription of Moon \& Dworetsky (1985). The use of the IUE spectrogrammes via the total flux yielded the same results: $\overline{\Delta T_{\text {eff }}}=\left(5 \pm \frac{150}{\sqrt{16}}\right) \mathrm{K}$ !

Once the temperature is given, surface acceleration and projected rotational velocity can be determined by matching theoretical line profiles based on Kurucz's fully line-blanketed model atmospheres to the observations.

\section{NGC 2264}

Figure 1 shows analysed objects of the young cluster NGC 2264, together with the ZAMS $(X=0.70, Z=0.03)$ from Hejlesen (1980). Since that particular cluster is very young, (age $<5 \cdot 10^{6} \mathrm{yrs}$ ) one expects that its stars would define 
an observational ZAMS, hopefully close to the theoretical one. This is not the case. Some cluster members deviate considerably from their expected position on the theoretical ZAMS. However, as the figure demonstrates, this occurs only for the fast rotators with $v \sin i \gtrsim 200 \frac{\mathrm{km}}{\mathrm{sec}}$. Thus we tried to estimate the rotational shift $\Delta T_{\text {eff }}, \Delta g$ (from $\Delta c_{0}, \Delta \beta$, taken from Collins \& Sonneborn) for each individual object in order to find the corresponding position of the nonrotating configuration. Firstly we assumed random orientation of the rotation axes, which corresponds to a mean inclination angle $\bar{i}=52^{\circ}$. This come close to $i=60^{\circ}$, one of the angles used by Collins \& Sonneborn (1977). With i fixed to $60^{\circ}$, we derived $w=\frac{\omega}{\omega_{\text {crit }}}=\frac{v_{\text {eq }}}{\omega_{\text {crit }}} \cdot R_{\text {eq }}(w)$ by iteration since the equator radius $R_{\text {eq }}$ depends explicitly on the rotation parameter $w$ itself (see Collins et al. 1991). $\omega_{\text {crit }}$, taken from the theoretical models, depends on mass and radius. With $w$ known, the expected displacements in surface acceleration and temperature could be estimated and corrected for. The result is given in Figure 2. All objects are now close to the theoretical ZAMS.
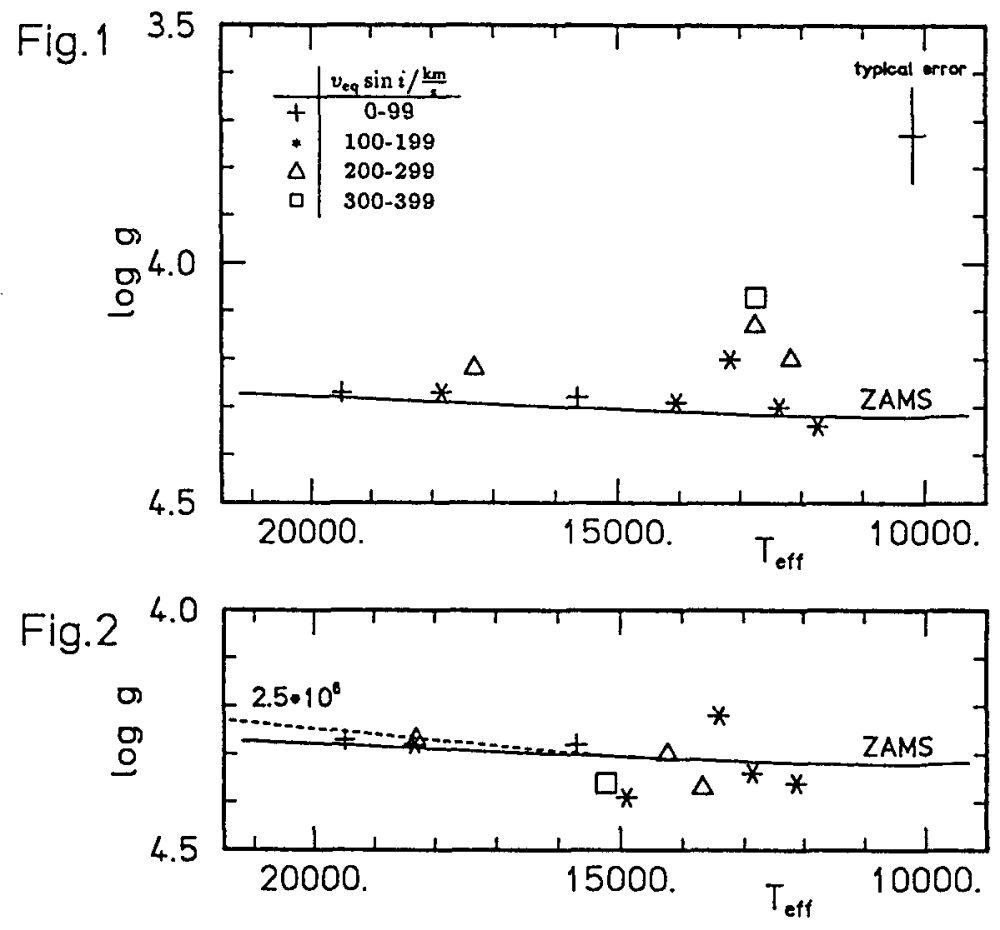

$\alpha$ Per

Figure 3 gives the $T_{\text {eff }}, g$ - plane for the $\alpha$ Per stars together with the appropiate isochrones from Maeder \& Meynet (1991) which include a moderate amount af overshooting. Figure 3 seems indeed to support the existence of two distinct populations, $\approx 6 \cdot 10^{7}$ and $2 \cdot 10^{8}$ yrs old, just as proposed by Eggen \& Iben (1988) and confirmed spectroscopically by Wolff (1990).

However, Figure 4 shows a different picture: There we have corrected for rotational displacements in the same manner as shown above for the NGC 2264 cluster, and one can now assigne one single cluster age $\left(5.5 \cdot 10^{7} \mathrm{yrs}\right)$. 
In conclusion, we found by means of accurate spectroscopic determination of stellar parameters and application of simple rotating stellar models:

- Fast rotation $(w>0.5)$ mimics evolution and widens the main sequence!

- Observed stellar parameters must be corrected for rotational effects before interpreting them!

- The rigidly rotating stellar models of Collins \& Sonneborn are sufficient for interpreting positions of rotating stars in the $\mathrm{H}-\mathrm{R}$ diagramme.

Fig. 3

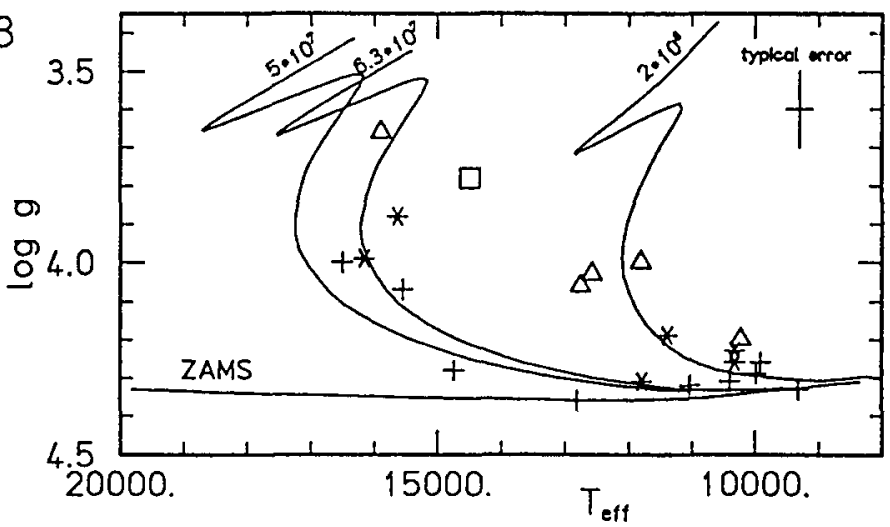

Fig. 4

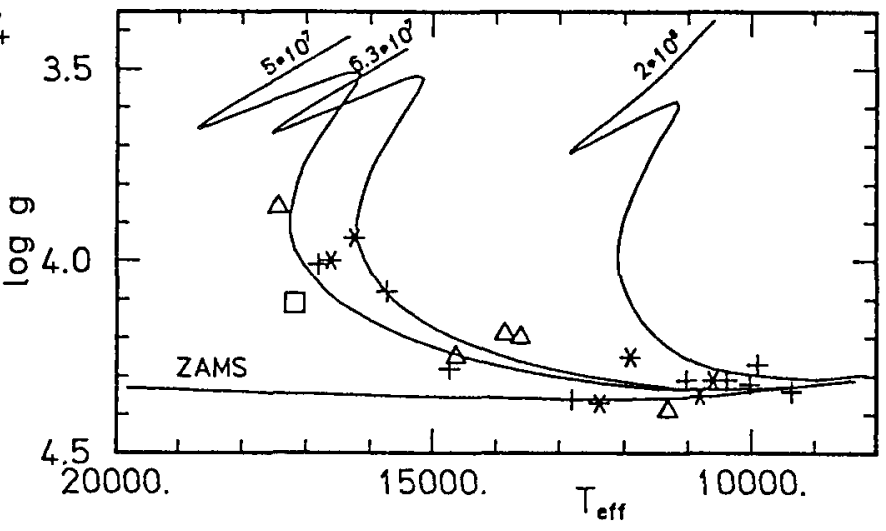

References

Collins, G.W., Sonneborn, G.: 1977, Astrophys. J. Suppl. 34, 41

Collins, G.W., Truax, R.J., Cranmer, S.R.: 1991, Astrophys. J. Suppl. 77, 541

Eggen, O.J., Iben, I.Jr.: 1988, Astron. J. 96, 635

Hejlesen, P.M.: 1980, Astron. Astrophys. Suppl. 39, 347

Maeder, A., Meynet, G.: 1991, Astron. Astrophys. Suppl. 89, 451

Moon, T.T., Dworetsky, M.M.: 1985, Monthly Not. Roy. Astron. Soc. 217, 305

Sackmann, I.-J., Anand, S.P.S.: 1970, Astrophys. J. 162, 105

Wenske, V.: 1992, Diplom Thesis, Christian Albrechts Universität, Kiel

Wolff, S.C.: 1990, Astron. J. 100, 1994 